Roberto Veraldi

UDC: 316.72

I'Università Gabriele d'Annunzio

DOI: 10.18485/dh.2015.2.ch2

di Chieti-Pescara

\title{
COMUNICAZIONE E SVILUPPO: PER UNA NUOVA DETERMINAZIONE SEMANTICA
}

\begin{abstract}
Summary
La comunicazione per lo sviluppo è un tema particolarmente attuale non solo per I'interesse dimostrato da organismi quale I'UNESCO o la FAO, ma anche per l'esigenza di rispondere ai criteri di sviluppo locale; cioè, maggiormente vicini al cittadino e al suo territorio. In altre parole, mentre è vero che la comunicazione da sola non può indurre lo sviluppo è anche vero che una comunicazione inadeguata può rallentare e rendere infruttuoso lo sviluppo ed impedire la partecipazione popolare
\end{abstract}

Parole chiave: Comunicazione, cultura, sviluppo locale.

Tutte le ricerche sulla funzione della comunicazione nelle politiche di sviluppo, confluite poi nel Rapporto MacBride del 1980, si concentrano su quella che venne definita la comunicazione per lo sviluppo: espressione che, pur non riferendosi alle regioni occidentali con economie in ritardo ma alle emergenze dei paesi del Terzo Mondo, è in grado di coprire le problematiche dei processi sociali basati sul dialogo, che mirano alla ricerca del cambiamento improntato allo sviluppo dell'interazione con il territorio.

Partiamo dal presupposto che il termine sviluppo è un termine polisemico. In maniera sintetica possiamo dare per accettata questa definizione:

Processo di cambiamento di strutture economiche; potenziamento delle capacità produttive che permette di avere una superiore quantità di beni e servizi rispetto al passato, cambiando le strutture socioeconomiche, i modelli culturali e le aspettative.

Dando per buona, per ora, questa definizione, la domanda che dobbiamo porci è: Si può parlare ancora di sviluppo locale, di localismo, di 
soggettività di un determinato territorio nell'era della globalizzazione dei mercati e del controllo planetario delle politiche di sviluppo?

Quello di sviluppo è un concetto fondamentale nell'ambito delle scienze sociali, attorno al quale si è approfondito il dibattito teorico. Tale dibattito, però, ha lasciato ancora aperte molte questioni; tra tutte il non aver portato ad una determinazione condivisa.

Potremmo aggiungere la formula

\section{SVILUPPO = CRESCITA MATERIALE + RAZIONALIZZAZIONE (OCCIDENTALE).}

Questa idea di sviluppo nasce da alcune visioni, fino a non molto tempo fa, fortemente dominanti e da alcune concezioni conseguenti:

Una forte convinzione evoluzionista che considera lo sviluppo un processo lineare, cumulativo, irreversibile;

Una forte convinzione industrialista, con una vocazione a replicare il modello occidentale di sviluppo socioeconomico;

Una forte convinzione etnocentrica, strettamente legata al secondo modello, in base alla quale la razionalità occidentale viene trasformata in un concetto di validità universale e allo stesso modo vengono considerati universali valori tipicamente occidentali come spirito d'impresa, profitto, sicurezza materiale, interesse personale.

Quello di sviluppo si presenta dunque storicamente come un concetto tipicamente e profondamente occidentale, in quanto ha avuto origine in Europa e si è diffuso in maniera globale, tanto che nella lingua di numerose culture extraeuropee non è facile rintracciare termini che rendano appieno il significato di sviluppo come noi lo intendiamo; inoltre, non si può non constatare che con la parola sviluppo si tende ad indicare l'universalizzazione dei benefici del Primo Mondo.

Forse il problema sta proprio qui: se da una parte lo sviluppo corrisponde alla modernità, identificata con i valori, le pratiche e le tecniche occidentali; dall'altra parte il sottosviluppo (il "fortunato" modello coniato dal Presidente degli Stati Uniti d'America, Harry Truman, con il lancio del "Punto IV" del programma di aiuti tecnici e finanziari ai paesi sottosviluppati, analizzato successivamente dallo studioso Walt Whitman Rostow nella sua famosa "Teoria degli Stadi"), corrisponde alla tradizione, ricondotta a valori, pratiche e tecniche differenti da quelle occidentali. 
È proprio in quest'ottica che la tradizione e le peculiarità locali, vengono intese come il principale ostacolo allo sviluppo. Infatti, come conseguenza logica, se si ritiene che il modello tecnico-culturale occidentale sia universale, qualcosa che sia altro da questo diventa un modello da scartare.

Questa prospettiva però risulta in parte fallace se pensiamo al caso concreto dell'economia asiatica che fa della tradizione, della cultura locale un punto di forza della sua crescita.

Pensiamo al caso delle Tigri Asiatiche, che hanno fatto della tradizione il loro punto di forza; oppure pensiamo a quelle molteplici attività "locali", che hanno fatto del rapporto dialettico con il tessuto sociale ed economico del passato, la riscoperta per una nuova economia sociale (pensiamo all'esempio dell' "Albergo Diffuso" che nasce come albergo orizzontale che si caratterizza per una serie di requisiti messi a punto dopo una lunga serie di esperienze sul campo, avviate a partire degli anni '80 in Friuli e in Sardegna e come avviene a Santo Stefano di Sessanio in Abruzzo, che rappresenta un nuovo modello di offerta turistica basato sul recupero dell'esistente e la valorizzazione delle risorse locali, seppur immerse nella modernità e interconnesse col mondo).

Ciò ci induce a pensare che lo sviluppo non deve essere inteso solo come una prospettiva economica, ribaltando il significato di crescita toutcourt, di cui solo gli economisti potevano e dovevano occuparsi.

E ritorno all'incipit. Lo sviluppo è un termine polisemico, ma ha nel suo stesso concetto l'idea profonda di sviluppo come emancipazione dell'uomo. Come riporta Marco Caselli: sviluppo delle persone, per le persone e dalle persone. In definitiva, lo sviluppo visto come un processo di ampliamento delle scelte della gente, una possibilità di condurre una vita lunga e sana, di acquisire conoscenze e di accedere alle risorse necessarie a un tenore di vita dignitoso. Per cui, formazione delle capacità umane e l'uso delle capacità acquisite. Proprio in base a ciò, il reddito diventa solo una delle opzioni che la gente vorrebbe avere ma che non rappresenta la somma totale della vita degli uomini. Lo sviluppo, quindi, deve essere qualcosa di più che la mera espansione del reddito e della ricchezza.

Il suo obiettivo deve essere l'individuo.

Se, pertanto, l'azione degli attori sociali è al centro dello sviluppo e se gli attori sociali sono riferibili alle società in cui esplicano le loro at- 
tività relazionali (quella costruzione di campo sociale di Pierre Bourdieu o quell'arena sociale, da me intesa come nuova costruzione semantica, in base alla quale ogni attore sociale razionalmente costruisce la realtà attraverso la sua relazione sociale con gli altri individui, aggiustandola 0 scomponendola di continuo, mutuando il meccanismo dalla teoria della strutturazione di Antony Giddens con la variante, proposta da me, che nelle realizzazioni delle pratiche sociali, le proprietà strutturali non "sono temporalmente presenti solo nel momento della costituzione dei sistemi sociali", perché se così fosse diventerebbero un'azione inintenzionale; al contrario, proprio perché attengono alla sfera umana/relazionale, sono connaturate alla socialità dell'individuo che, trasformando le sue azioni in azioni di individualismo sociale, vanno ad incidere sulla realizzazione dell'intera realtà sociale che, così, diventa una conseguenza razionale ed empirica della sua naturale propensione alla ingegneria sociale della realtà che diventa visibile, questo si, solo al momento della costruzione plastica di nuove oggettività, ma che è sempre presente nelle attività quotidiane a prescindere dalla messa in pratica o meno di comportamenti generativi di nuovi "fatti sociali"), realizzando di fatto nuove conseguenze sociali e adattandole in continuazione ai cambiamenti in atto; allora il problema diventerà quello di una nuova definizione di sviluppo: potremmo forse parlare di sviluppo coalizionale come momento di raccordo e di sintesi tra capacità relazionali, capacità economiche e le capacità culturali intese come valorizzazione della tradizione; dove, però, lo sviluppo non ricade nello sviluppismo espressione cara a Latouche che indica come l'idea dello sviluppo sia ormai un'azione tossica e una scelta letale per le nuove generazioni se ciò non porta ad un ripensamento del concetto stesso, oltre a indicare anche che "è l'idea stessa di sviluppo che sta sparendo dallo scenario come conseguenza diretta del genio occidentale moderno, anch'esso in declino, secondo il quale il progresso scientifico e tecnologico migliorerebbero necessariamente e inevitabilmente le condizioni di vita dei popoli della terra", non è uno sviluppo locale come ossimoro che entra in contrasto con la peculiarità della crescita come consumo e il locale come attenzione verso la tradizione e la sostenibilità, ma uno sviluppo (coalizionale, appunto) che produce movimenti circolari di saperi, persone, economie diverse tra loro e tra loro meticciate dando ad ognuna di esse una pari dignità e giungendo a nuove costruzioni di realtà sociali, con 
ricadute anche plastiche visibili e tangibili, aventi tutte una loro dignità e una prospettiva storico-sociale nel panorama globale.

Ciò implica anche una Govern-Azione della globalizzazione ed una efficace comunicazione dei fenomeni in atto per una realizzazione della giusta considerazione territoriale dell'arena sociale di riferimento, per evitare che l'interconnessione tra reti locali si trasformi in una concatenazione tra realtà sociali dominanti versus società dominate; con la conseguenza di una sorta di diminuzione di sovranità nazionale delle realtà più deboli, dimenticando che tutte le società hanno, per la loro stessa natura, uguale parità e dignità all'interno di quella nuova arena sociale.

Nel corso degli ultimi anni, infatti, il fenomeno della globalizzazione si è imposto all'attenzione di tutti. La globalizzazione in presenza di profondi cambiamenti che non trovano spiegazioni nelle tradizionali ideologie, ha finito per acquisire un significato paradigmatico che dovrebbe spiegare qualsiasi fatto o evento che succeda in politica ed in economia. In definitiva, si vuole porre in analisi l'esistenza di uno spazio (e di uno strumento efficace connesso), nel quale i sistemi locali possano affermare una loro identità ed una loro capacità di crescita.

Certamente, se si dovessero accettare in maniera troppo supina quelle interpretazioni della globalizzazione che vedono il fenomeno come una minaccia per le identità locali, le speranze per una crescita autonoma delle realtà sociali locali sarebbero di sicuro destinate a suscitare molte perplessità; infatti, se diamo corpo a queste paure il sistema socio-economico messo in piedi a livello mondiale renderebbe, nelle evidenze empiriche è dimostrato, impossibile la presa di coscienza dei territori locali e la messa in pratica di sistemi di nuove relazioni socioeconomiche tra i territori stessi, seppur inseriti in ambiti globali (sempre intesi come interconnessione di reti locali di fattori situati).

Per cui dobbiamo capire se questo rimane uno scenario unico oppure un finale di film aperto a più soluzioni.

Da questo punto di vista, l'aspetto più borderline rimane quello di un tentativo, voluto dal Sistema-Mondo inteso come razionalizzazione della realtà economica, di deresponsabilizzare le realtà locali attraverso atteggiamenti programmati di delega verso i decisori "altri" che non rappresentano e non sono l'espressione delle realtà locali e soprattutto non 
sono a conoscenza delle peculiarità e delle vocazioni del "locale". Al contrario, l'atto cosciente di autotutela e di ripresa del sé del mondo micro verso il mondo macro, pone in atto una presa di autorevolezza del locale nei confronti del globale, che ha nella sua espressione più fortunata (la Glocalizzazione), il nesso più forte. Rapporto che rimette in equilibrio il Sistema delle relazioni sociali. Per cui, globale non Vs locale, ma risposta congiunta ai cambiamenti in atto per la valorizzazione di quelle arene sociali che danno la misura della società nel suo complesso.

Per converso, però, dobbiamo tenere in buona considerazione la lezione di Serge Latouche, quando afferma che la mondializzazione dell'economia [con i suoi risvolti negativi, N.d.A], si realizza pienamente solo con la trasformazione di tutti gli aspetti della vita in questioni economiche, se non in merci. [...] la mondializzazione è di fatto anche tecnologica e culturale, e copre la totalità del pianeta.

Il problema, allora, sarà quello di mettere in piedi strategie di autogoverno delle autocostruzioni di senso delle autonomie locali che dovranno sfociare in attività di promozione dello sviluppo locale (da me inteso come Sviluppo Coalizionale, in cui I'Individualismo Sociale trova applicazione e sintesi. Individualismo sociale come momento di superamento tra Olismo ed Individualismo; nel caso di specie, come momento di adattamento tra Stato e Mercato e come luogo di raccordo tra Stato-Economia-Mercato per la creazione di nuovi modelli sociali che si trasformano, così, in luoghi della quotidianità che, però, dovranno essere comunicati per poter essere condivisi e accettati).

Infatti, i cittadini socializzano e si riconoscono come parte attiva di una comunità attraverso la memoria dei luoghi e attraverso il racconto dei luoghi. "Una società", secondo la definizione di E. Durkheim, "non è costituita semplicemente dall'insieme degli individui che la compongono, dal terreno che essi occupano, dalle cose di cui si servono, ma è costituita in primo luogo dall'idea che essa si forma di sé".

I luoghi non sono oggetti immutabili, sono astrazioni, si scompongono, si ri-aggregano in altre forme, i confini sono mobili, in altri termini: invecchiano, si trasformano, muoiono, rinascono. Insieme ai luoghi, invecchiano anche i loro racconti. Un racconto del territorio è vecchio quando ne banalizza l'esperienza, quando le linee di fuga sono spezzate, quando non contiene la possibilità di immaginarne un futuro. 
E' quello che sembrano sperimentare molti territori oggi nel nostro paese, costretti in racconti scolastici ed in confini locali tracciati prima dell'avvento della mobilità di massa, quando la stragrande maggioranza degli italiani andava a lavorare, faceva la spesa, accompagnava i figli a scuola a piedi, in bicicletta, a dorso di mulo o su treni molto più lenti degli attuali. Deve diventare il racconto dei territori viventi: narrare per conoscersi e narrare per competere.

Deve trovare, pertanto, quel legame concreto che, nella totalità di un fatto storico-sociale, costituisce il particolare elemento di coesione fra un fatto ideologico e il portato sociale (ovvero che spieghi, sociologicamente, la dinamica culturale interna; in altre parole, la cultura assimilata, vissuta e comunicata per poi farsi tratto unificante e ideologicamente fondante di nuove realtà sociali), anche perché diventa fondante il rapporto tra presente "e il suo passato, come risorsa di, o minaccia per, all'interno di una visione di società che sempre più mira al futuro, al controllo ed alla pianificazione su base razionale del cambiamento (il progresso)".

Se la ricerca sociale ed economica si occupa di territori viventi (come afferma Tantillo), deve, per me, porre necessariamente I'Individualismo Sociale e lo Sviluppo Coalizionale al centro delle sue riflessioni e dei suoi racconti. Deve cambiare la prospettiva: dare rilevanza anche al patrimonio della cultura immateriale e a quella connessa alle attività, perché queste attività (così come le ho intese in precedenza), sono produttrici di economia o generative di società, attraverso i loro portati di senso materiale e immateriale.

Con una società in frantumi, in condizioni di precarietà e vittima di un grave indebolimento territoriale sul quale incidono sia la diminuzione dei servizi alle persone e alle imprese sia il peggioramento delle condizioni strutturali della popolazione, sia la polverizzazione della struttura commerciale e i bassi livelli di produttività occorrono nuove traiettorie di riflessione e di ingegneria sociale, ispirate ad una sorta di controesodo culturale che ridarebbe respiro alla centralità del territorio e ai fattori in esso situati. Alla base di questa inversione di tendenza ci sarebbe sia il desiderio di costruirsi una diversa qualità della vita, ma anche la volontà di riappropriarsi di luoghi percepiti a volte come quelli delle origini. 
Allora dobbiamo reinventarci il racconto, reinventare le formule del racconto, reinventare le modalità per una efficace attività di comunicazione dei territori e delle comunità locali, all'interno dello scenario appena tracciato di uno sviluppo coalizionale (già spiegato come sintesi tra capacità relazionali, capacità economiche e le capacità culturali intese come valorizzazione della tradizione). In sintesi estrema, un'altra idea di sviluppo; oppure un'idea alternativa allo sviluppo così come è impostato ora e che, pertanto, rispecchi una discontinuità con le definizioni semantiche del passato e con le spiegazioni contorte dell'economie del presente; per cui, attraverso un richiamo forte alla creatività delle comunità locali (dove sono forti, in senso paretiano, gli istinti delle combinazioni), si deve giungere alla creazione di una nuova strategia socioeconomica mediante una operazione di decostruzione dell'immaginario economico che passi necessariamente da nuove forme del comunicare l'economia stessa come una economia universale; essa, a mio avviso, rappresenta al contrario una costruzione culturale e storica: ciò implica che è possibile cambiarla. Tale cambiamento, deve, però passare attraverso lo strappo della supremazia di una sola disciplina (in questo senso, quanto affermava Pierre Bourdieu parlando di economia e di sociologia, dichiarando che ambedue analizzano i prodotti di una costruzione sociale, di modo che non è possibile descrivere adeguatamente i processi economici senza fare riferimento alla sociologia. Invece di opporle, come avviene tradizionalmente, è il momento di rendersi conto che sociologia ed economia costituiscono in realtà una unica e identica disciplina che ha come oggetto l'analisi dei fatti sociali, dei quali le transazioni economiche rappresentano dopotutto solo un aspetto), ed aprirsi alla condivisione tra le discipline mediante l'invito ad una riflessione su tematiche che stanno investendo già da tempo i nostri modelli socioeconomici e ne stanno per costruire di nuovi: il tentativo è quello di rendere un valore "la rottura generata dal crepuscolo dell'economia, che si manifesta con l'esaurimento delle significazioni immaginarie fondatrici dell' epoche economica. Uscire dall'economia [da questo genere di economia non fondata sull'etica economica e non fondata sull'individuo/sulla Persona come proprietario della moneta in quanto titolare di diritti soggettivi e, tra questi, una nuova formulazione del reddito di cittadinanza, N.d.A], significa chiaramente uscire dal capitalismo e rompere con l'occidentalizzazione del mondo" anche attraverso compromessi e ibridazioni. 
Assume, però, anche un altro significato: riprendere nelle proprie mani i destini del proprio territorio, ridando voce agli emarginati, ridando speranze agli esclusi, ridisegnando i confini culturali e spaziali attraverso la messa in pratica e la condivisione di nuovi contesti sociali dell'agire comunicativo i cui protagonisti, pur con i limiti propri della società complessa, si dimostrano in grado di farsi portavoce di una revisione critica delle proprie tradizioni di riferimento.

\section{Bibliografia essenziale di riferimento}

AA.VV, Comunicazione e società oggi e domani. Il rapporto MacBride sui problemi della comunicazione nel mondo, RAI-ERI Edizioni, Roma, 1982

G. Auriti, Il paese dell'utopia, Solfanelli Editore, Chieti, 2002.

K. Besancon, The Collapsing Vision of Global Development (conferenza del PNUD, Bucarest, Settembre, 1992).

A. Bianco, Introduzione alla Sociologia dello sviluppo, FrancoAngeli, Milano, 2007. P. Bourdieu, Le strutture sociali dell'economia, Asterios Editore, Trieste, 2004.

M.Caselli, Misurare lo sviluppo. Tecniche e problemi, ECIG, Genova, 2001.

G. Dall'Ara (, a cura di), Come progettare un piano di sviluppo turistico territoriale, Halley Editore, Matelica, 2009.

E. Durkheim, Le forme elementari della vita religiosa, Edizioni di Comunità, Milano, 1982.

M. Ghisleni, \& W. Privitera (a cura di), Sociologie contemporanee. Bauman, Beck, Bourdieu, Giddens, Touraine, UTET, Novara, 2009.

S.Latouche, L'invenzione dell'economia, Bollati Boringhieri, Torino, 2005.

S. Latouche, Come sopravvivere allo sviluppo, Bollati Boringhieri, Torino, 2009.

S. Latouche, La fine del sogno occidentale. Saggio sull'americanizzazione del mondo, Elèuthera Edizioni, Milano, 2010.

P.Malizia, Lezioni di sociologia della cultura, Aracne, Roma, 1999.

P.Malizia, Comunic-a-zioni. Strutture e contesti sociali dell'agire comunicativo, FrancoAngeli, Milano, 2006.

F. Tantillo, Abruzzo Reset: un progetto sperimentale per la conoscenza e la comunicazione del territorio, in Tafter Journal, $\mathrm{n}^{\circ} 4$ - Maggio 2008.

UNDP, Rapporto su lo sviluppo umano. Come ridurre le diseguaglianze mondiali, Rosenberg \& Sellier, Torino, 1993a.

R. Veraldi, Globalizzazione e società locale, Edizioni Universitarie Romane, Roma,2007.

R. Veraldi, Manuario. Work in progress per temi sociologici, Aracne, Roma, 2007.

R. Veraldi (a cura di), Etica-Economia_Società. Sistemi sociali ed economici in transizione, Edizioni Universitarie Romane, Roma, 2009. 


\title{
Roberto Veraldi
}

University of Gabriele d'Annunzio of Chieti-Pescara

\section{COMMUNICATION AND DEVELOPMENT: FOR A NEW SEMANTIC RESOLUTION}

\begin{abstract}
Summary
Communication for development is a particularly current subject, not only for the interest given by organizations such as UNESCO and FAO, but also the need to respond to the criteria of local development, i.e., a great deal nearer to the citizen and his territory. In other words, while it is true that communication alone cannot bring about development, it is also true that inadequate communication can slow down and make development fruitless, besides encumbering people's participation.
\end{abstract}

Key words: Communication, culture, local development 